\title{
LOS EXCERPTA DE BEDA (DE TEMPORUM RATIONE 19 Y 23) EN RIPOLL (ACA, RIPOLL 59 Y VAT. REG. LAT. 123) ${ }^{1}$
}

The analysis of texts and glossaries contained in the manuscript ACA Ripoll 59 reveals various compilations of glossaries and "computation» texts, besides Priscian's grammar, which is the most important text in the manuscript. The analysis and identification of the fragments in ACA Ripoll 59 the same as those in Vat. Reg. Lat. 123, which also belongs to the manuscripts found in Ripoll, corresponding to various chapters of Beda, allow us to study the different currents that exerted their cultural influence in the Monastery of Santa Maria de Ripoll. Through the variants stablished for each of the texts that have bean identified, one detects the influence of various families of manuscripts in the composition of these fragments.

\section{INTRODUCCIÓN}

El manuscrito del Archivo de la Corona de Aragón, fondo del monasterio de Ripoll, número 59 (Riu $=$ Riuipullensis), es un códice en pergamino, con unas medidas de $310 \times 225 \mathrm{~mm}$, escrito en Ripoll alrededor del año mil, con una escritura minúscula carolina ${ }^{2}$. El ms. contiene el texto correspondiente a la Gramática de Prisciano (ff. 2-288v) ${ }^{3}$, además de tres glosarios: el primero de ellos ocupa los ff. $289 \mathrm{v}-295 \mathrm{v}$; el segundo, ordenado alfabéticamente, em-

1 Este trabajo forma parte del Proyecto de Investigación concedido por la DGICYT PS89-055 y del Programa AIRE 94/1.

${ }^{2}$ Pueden consultarse los siguientes trabajos, para recabar una más completa información sobre el ms.: R. Beer, Die Handschriften des Klosters Sta. Maria de Ripoll, en traducción catalana de P. Barnils, Los manuscrits del monestir de Santa Maria de Ripoll, Barcelona 1909, p. 53; R: Beer - Z. Garcia Villada, Bibliotheca Patrum Latinorum Hispanienses, Bd. II: Barcelona-Ripoll, Viena 1915, p. 96 (existe una revisión de este catálogo, llevada a cabo por F. Miquel Rossell y M. D. Mateu Ibars en el ACA desde 1973, inédita, donde aparece una breve descripción de nuestro ms.). También puede consultarse el catálogo de Próspero de Bofarull (que fuera director del ACA), publicado por F. Valls i Taberner con el título de Códices manuscritos de Ripoll, Madrid 1931, p. 64; la monografia de J. M. Millàs i Villacrosa, Assaig de les idees fisiques i matemátiques a la Catalunya medieval, Barcelona $1983(=1931)$, pp. 237-238.

${ }^{3}$ Fol. 2: Incipit ars Prisciani uidi (sic) discreticimi grammatici. 
pieza en el f. $295 \mathrm{v}$ con $A$ ssida-lucida y termina en el f. $299 \mathrm{r}$ con Trias: trinitas; el tercer glosario, sin ningún tipo de orden, mezcla glosas cortas con otras muy largas, que beben de las Etymologiae de Isidoro ${ }^{4}$.

El manuscrito Reg. Lat. 123 de la Biblioteca Apostolica Vaticana, de probable procedencia ripollesa, es un códice en pergamino, con unas medidas de $360 \times 280 \mathrm{~mm}$., escrito en el año 1056 , con una escritura minúscula carolina. Se trata de un manuscrito misceláneo, principalmente de carácter astronómico y cosmográfico, que reúne excerpta de distintos autores (Plinio, Higinio, Macrobio, Isidoro, Beda, etc.), ordenados en cuatros libros: De sole, De luna, De natura rerum y De astronomia.

En el presente trabajo ${ }^{5}$ estudiaremos tres fragmento de Riu. (los ff. $303 \mathrm{~V}$ 304r) y uno de Reg. Lat. (los ff. 5r-6r) que hemos identificado con diversos textos de Beda.

Los dos primeros fragmentos de Riu. (ff. 303v-304r) corresponden a los capítulos XIX y XXIII del De temporum ratione ${ }^{6}$ de Beda; el tercero (ff. $302 v-303 r$ ) corresponde al capítulo VIII del De diuisionibus temporum ${ }^{7}$ atribuido a Beda.

Podremos sugerir, de esta forma, la probable relación de los mss. ripolleses con los principales mss. bedanos, para intentar saber de dónde procede esa fuente de conocimientos en Ripoll (sobre todo, a través de las variantes de Riu. respecto de Jones $^{8}$ documentadas en otros mss. y contempladas en el aparato crítico). Podremos conocer mejor el texto en sí mismo y encuadrar los mss. que lo contienen en alguna de las corrientes manuscritas de Beda en Europa, lo cual nos puede permitir, a su vez, entrever las posibles relaciones del scriptorium de Ripoll con otros centros europeos de la época ${ }^{9}$.

\footnotetext{
${ }^{4}$ Véase el estudio de J. Llauró, «Los glosarios de Ripoll», Analecta Sacra Tarraconensia III, 1927, pp. 331-389, espec., pp. 364-388, en el que se recogen solamente las glosas contenidas en los ff. 289r-295v; cabe señalar que la numeración dada por Llauró no coincide con la numeración que presenta actualmente dicho manuscrito.

Una parte de la tesis doctora de Gemma Puigvert se centra en la edición y estudio de las glosas científicas de este manuscrito.

${ }_{5}^{5}$ Basamos nuestros datos sobre la lectura directa del ms. Riu. y del ms. Reg. Lat.; para el resto de mss. seguimos la edičión de Jones.

${ }^{6}$ Cf. Bedae Venerabilis Opera, De Temporum Ratione Liber. Ed. de Ch. W. Jones, Corpus Christianorum. Series Latina, CXXIIIB, Turnholt 1977.

${ }^{7}$ Cf. Migne, P.L., XC, cols. 656-657. Véase también Ch. W. Jones, Bedae Pseudepigrapha: Scientific Writings Falsely Attributed to Bede, Oxford University Press 1939, pp. $48-51$.

${ }^{8}$ Nos referimos a la edición del De Temporum Ratione de Beda realizada por Ch. W. Jones, publicada en el Corpus Christianorum. Series Latina, CXXIIIB, Turnholł 1977.

${ }^{9}$ Ofrecemos como apéndice el capítulo VIII: $\mathrm{De}$ die del De diuisionibus temporum incluido, junto a los demás capítulos que integran esta obra, en el ms. Reg. Lat., lo cual nos permite relacionar los dos mss. ripolleses.
} 


\section{CONSTITUCIÓN DEL APARATO DE VARIANTES}

Tomamos como texto de referencia para detectar las posibles variantes de $R i u$. respecto del DTR establecido, el de la última edición del DTR realizada por Jones.

Los mss. que Jones utiliza para su edición son los siguientes:

1. $B=$ Berlin, BDDR, 128 (Phill. 1831) (ca. A.D., 800, Verona), foll. 16r$89 \mathrm{v}$.

2. $\mathrm{C}=$ Cologne, DB, 103 (ante A.D. 8190 , Cologne), foll. 52v-184r.

3. $\mathrm{F}=\mathrm{Kassel}$, LB, Astron. fol. 2 (A.D. 814? Fulda), foll. 10r-82v.

4. $\mathrm{K}=$ Karlsruhe, LB, Augiensis 167 (s. IX1, Reichenau?), foll. 23v-64r.

5. $\mathrm{L}=$ London, BM, Cotton Vesp. B 6 (s. IX, $\mathrm{n}$. France), foll. 1r-102r.

6. $\mathrm{M}=$ Melk, SB, 370, olim G. 32 (s. IX, Auxerre), pp. 59-191.

7. $\mathrm{Mu}=$ München, SB, Clm 14725 (s. IX' ${ }^{1}$, n. France), foll. $25 \mathrm{v}-166 \mathrm{v}$.

8. $\mathrm{S}=$ St. Gall, SB, 251 (s. IX ${ }^{1}$, St. Gall), pp. 45-181.

Rarius citantur:

9. $A=$ Paris, BN, N.A. 1632 (s. IX, Fleury), foll. 10r-67v.

10. $\mathrm{E}=$ Genève, $\mathrm{BY}, 50$ (s. IX $\mathrm{X}^{1}$, Massai), foll. 45r-120r.

11. $\mathrm{H}=$ Paris, $\mathrm{BN}$, lat. 13403 (s. IX, Corbie), foll. $1 \mathrm{v}-110 \mathrm{r}$.

12. $\mathrm{N}=$ Milano, BA. D 30 Inf. (s. IX', Bobbio?), foll. 23r-121r.

13. $\mathrm{P}=$ Paris, $\mathrm{BN}$, lat. 7296 (s. IX $\mathrm{X}^{\mathrm{l}}$ ), foll. $1 \mathrm{r}-111 \mathrm{r}$.

14. $\mathrm{R}=$ London, $\mathrm{BM}$, Regius $15 \mathrm{~B}$ XIX (s. IX $\mathrm{X}^{2}$, Reims), foll. 38r-78v.

15. $\mathrm{T}=$ Berlin, BDDR, 131 (Phill. 1869) (s. IX', Trier), foll. 15r-139.

Para su análisis ofrecemos la lectura de Riu. con indicación de folio; a continuación, y por capítulos, la lectura variante que ofrece Jones, con indicación de la línea de edición, y entre paréntesis, la lectura de los mss. coincidentes, cuando ésta exista, con Riu. Todas las variantes, señaladas específicamente en cursiva, llevan su propio número de inventario al que nos remitiremos en nuestro análisis posterior.

Capitulo XIX.

1. f. $303 \mathrm{v}$, Si quis = lín. 2, Si qui (si quis $C M u^{2} S^{2}$ ).

2. f. $303 \mathrm{v}$, querit inueniet $=$ lín. 4 , quaerit inueniat (inueniet $B$ ).

3. f. $303 \mathrm{v}$, Siquidem totum $=$ lín. 4 , Siquidem totam (totum $B S^{1}$ ).

4. f. $303 \mathrm{v}$, seriem que de XII mensibus continetur = lín. 5, seriem quae duodecim mensibus continetur (de XII $B$ ).

5. f. $303 \mathrm{v}$, Simul et mensium signorumque $=$ lín. 13, Simul et mensium singulorum signorumque (singulorum om. $B$ ).

6. f. $303 \mathrm{v}$, mensium retro adnotatis $=$ lín. 16 , mensium retro adnotatas (-tatis $C^{2} L^{2} M P^{2} R S^{2}$ ). 
7. f. $303 \mathrm{v}$, in latitudinem lineis $=$ lin. 18 , in latitudine lineas (-dinem lineis $B)$.

8. f. $303 \mathrm{v}$, habet scire $=$ lín. 21 , habeat scire (habet $B$ ).

9. f. $303 \mathrm{v}$, antea retro inspicients $=$ lín. 24 , ante ac retro inspiciens (antea retro $B$ ).

10. f. $303 \mathrm{v}$, aliquod quo a cetera $=$ lín. 26 , aliquod quod et caetera (quo $B C F)$.

11. f. $303 \mathrm{v}$, initia deprehendis esse $=$ lín. 31 , initia deprehendes esse (-dis $B C S)$.

12. f. $303 \mathrm{v}$, crescente seu decrescente $=$ lín. 34 , crescentem seu decrescentem (crescente $B$ ).

Capítulo XXIII.

13. f. $304 \mathrm{r}$, cursum lunae = lín. 3 , lunae cursum (cursum lunae $S$ ).

14. f. $304 \mathrm{r}$, simili modo notata $=$ lín. 7 , simili modo notatam (notata $B$ ).

15. f. $304 \mathrm{r}$, non desinet $=$ lín. 7 , non desinit (-net $B L^{1} R$ ).

16. f. $304 \mathrm{r}$, utrumque litteris = lín. 16, utrinque literis (utrumque $M u$ ).

\section{ANÁLISIS DEL APARATO DE VARIANTES}

El corpus de variantes que hemos establecido permite relacionar Riv. con alguna de las corrientes manuscritas de Beda en Europa, para tener algunos indicios del lugar de procedencia del texto bedano que se leía en Ripoll y, por tanto, ir avanzando en el conocimiento de las relaciones de Ripoll con otros centros o zonas geográficas.

En este caso, nos parecen significativas las aproximaciones de las variantes de Riu. respecto del texto de Jones, en el que encontramos lecturas paralelas en algunas de las familias de los mss. citados por este último. Una gran mayoría de las variantes de Riu. coinciden con el ms. $B$, tal como se deduce del estudio siguiente:

A. Variantes de Riu. coincidentes con el ms. $1=B$. Números $2,3,4,5,7$, $8,9,10,11,12,14,15$.

B. Variantes de Riu. coincidentes con el ms. $2=C$. Números $1,6,10,11$.

C. Variantes de Riu. coincidentes con el ms. $3=F$. 0 .

D. Variantes de Riu. coincidentes con el ms. $5=L$. Números 6,15 .

E. Variantes de Riu. coincidentes con el ms. $6=M$. Números 6 .

F. Variantes de Riu. coincidentes con el ms. $7=M u$. Números 1, 16.

G. Variantes de Riu. coincidentes con el ms. $8=S$. Números 1, 3, 6, 11, 13.

H. Variantes de Riu. coincidentes con el ms. $13=P$. Números 6 .

I. Variantes de Riu. coincidentes con el ms. $14=R$. Números 6,15 . 
A partir de la agrupación de mss. afines (nunca se pretende hacer un stem$m a$ ), de la que Jones habla ( $c f$. pp. 241-242), se pueden establecer los siguientes grupos o familias:

1. En un primer grupo 1, formado por dos subgrupos: 1) mss. 1, 10;2) 3, 12 , podrían englobarse las variantes que hemos listado con las letras $A(=12$ variantes); no existe ninguna variante de Riu. coincidente con los mss. 3, 10 y 12 . Son, en total, 12 variantes de Riu. que coinciden con lecturas de mss. de este grupo. De entre éstos, las más numerosas son las del grupo A $(=12)^{10}$.

El ms.1 (ms. Berlin, BDDR, 128 (Phill. 1831) (ca. A.D. 800, a Verona) pertenece, como hemos visto, al subgrupo 1, dentro del grupo 1, que Jones describe como: «a group, evidently centering in Germany, is marked by transposition of capitula and chapters, especially chapters II and III, VI and VII, LIV and LV» 1 !

2. En su segundo grupo 2, formado también por dos grupos: 1) 13,$11 ; 2$ ) 2, 6; este segundo subgrupo está formado, a su vez, por otro subgrupo $2_{a}$ ) 15 , $2,6,7$. Podrían englobarse las variantes que hemos listado con las letras $\mathrm{H}$ (= 1 variante); $\mathrm{B}$ (=4 variantes); $\mathrm{E}$ ( $=1$ variante); $\mathrm{F}$ ( $=2$ variantes) Son, en total, 8 variantes (frente a las 12 del grupo anterior). Este grupo, en palabras de Jones, «has two subgroups: (1) texts retaining one or more indications of an exemplar (in a codex without DT and DNR) in which chapter XV was omited. (2) Texts very like $\mathrm{C}$ and $\mathrm{M}$ from which the Laon Commentary below is transcribed. These texts often contain glosses which interlink with the Laon glosses, as in a special subgroup ( $2_{\mathrm{a}}$ ) of very similar MSS. The other identifiable members of subgroup 2 are...» ${ }^{12}$.

3. Un tercer grupo 3, formado por otros mss. que no han sido cotejados en la edición y que, per este motivo, no han sido tenidos en cuenta.

Vemos, pues, cómo desde el punto de vista cuantitativo, Riu. apunta hacia el primer gran grupo, y, dentro de éste, al primer subgrupo.

\section{IV, CONClusiones}

Del corpus de variantes establecido (las de Riu. respecto del texto de Jones documentadas por otros mss.) se desprende una clara relación de Riu. con los mss. del grupo 1 y de entre éstos, con el ms. 1., un ms. veronés, pero con un arquetipo (no muy lejano) claramente insular (¿quizá de Bobbio?), tal y como

10 La mayor coincidencia de variantes de Riu. con el ms. 1 se observa también en el estudio realizado por J. Gómez Pallarès, «Los excerpta de Beda (De Temporum Ratione, 25-35) en el ms. ACA, Ripoll 225», EMERITA 59, 1991, pp. 101-122.

11 Op. cit., p. 241.

12 Op. cit., p. 241. 
demuestran las frecuentes abreviaturas insulares que posee. Así pues, podemos precisar que el texto de Beda conocido en Ripoll procede, en última instancia, de la zona geográfica de Verona, en la que, en su momento (principios del siglo IX, siglo X-inicios del XI), pudieron darse influencias y contactos entre el monasterior catalán y la zona mencionada.

Ésta sería una primera vía de recepción del texto; de una segunda vía (franja geográfica que uniría Colonia con Auxerre) parten los excerpta de Beda (DTR, 25-35) ${ }^{13}$ contenidos en el ms. ACA, Ripoll 225, así como los diversos excerpta de Beda (DTR, 27 y 34; DNR, 5-7; 9; 11-20;22-23) contenidos en el Liber. de astronomia del ms. Vat. Reg. Lat. $123^{14 .}$

Apéndice. Edición del capítulo VIII: De die de la obra De diuisionibus temporum atribuida a Beda (a partir de los mss. Riu. 59, ff. 302v-303r y Reg. Lat. 123, ff. 5r-6r).

Beda (?), DDT, VIII.

- Quomodo dies in tribus principalibus linguis uocatur?

- Ita: ella in Hebraea '; emera in Greca; dies in Latina.

- Quis primitus diem nominauit?

- Deus, ut in Genesi dicitur: Apellauit lucem diem et tenebras uocauit noctem.

M. Dies unde nomen accepit?

M. A diuisione, eo quod diuidat lucem a tenebris. Siue dies dicti sunt a diis, quorum nomina Romani quibusdam sideribus sacrauerunt. Item dies dicitur eo quod lucem et tenebras disiungat ac diuidat, et appellatiue dicitur dux operum, et absentia tenebrarum. Ema in Hebraeo, hemera in Graeco, dies in Latino.

13 Véase el trabajo del Prof. Joan Gómez Pallarès, op. cit., pp. 121-122.

14 El estudio de este ms. forma parte también de la tesis doctoral de Gemma Puigvert.

1 Ebrea, Riu. 59. 
— Vnde dies nomen accepit? ${ }^{2}$

- Isidorus dixit: Dies est dux operum et absentia tenebrarum solisque orientis presentia; est autem dicta quia lucem a tenebris discernit atque diuidit. Ita dies diuidendo lucem a tenebris dicta uel eo quod iucundus ${ }^{3}$ sit diis et incerti generis ${ }^{4}$ inter masculinum et femininum, ut gramaticus dixit: Dies incertum nomen inter masculinum et femininum. Ideo nomen masculini quod inuenitur festi dies, et in aduerbio hodie, quasi hoc die. Ideo nomen femenini ${ }^{5}$ quia nomen quinte declinationis et diminutiuum eius diecula inuenitur.

— Quomodo diffinitur ${ }^{6}$ artificalis ${ }^{7}$ dies? ${ }^{8}$

- Isidorus dixit: Dies est solis orientis praesentia, quousque ad occasum perueniet.
D. Dies unde nomen accepit?

M. A diuisione, eo quod diuidat lucem a tenebris. Siue dies dicti sunt a diis, quorum nomina Romani quiibusdam sideribus sacrauerunt. Item dies dicitur eo quod lucem et tenebras disiungat ac diuidat, et appellatiue dicitur dux operum, et absentia tenebrarum. Ema in Hebraeo, hemera in Graeco, dies in Latino.

D. Quis est ergo ille dies artificialis? M. Ab ortu solis usque ad occasum, dies artificialis dicitur, id est, praesentia solis super terram. Vnde Isidorus dicit: Dies, praesentia solis est, siue sol super terram: sicut nox dicitur absentia solis, id est, sol sub terris. Diffinitio diei bifarie diuiditur, hoc est uulgariter, id est, abusiue; et naturaliter, id est, proprie.

\footnotetext{
2 Cf. Isidoro, Etym., V, XXX: Dies est praesentia solis, siue sol supra terras, sicut nox sol sub terris.

Cf. Isidoro, DNR, I, 1: Dies est solis orientis praesentia, quousque ad occasum perueniat.

Como se puede comprobar, nuestros mss. presentan como dos respuestas distintas el contenido que se ofrece en la respuesta a la primera cuestión formulada en el De diuisionibus temporum.

3 iocundus Vat. Reg. Lat. 123.

4 ieneris Riu. 59.

5 feminini Vat. Reg. Lat. 123.

6 difinitur Riu. 59.

7 articularis Vat. Reg. Lat. 123.

8 Cf. Isidoro, Etym., V, XXX, 1: Dies est praesentia solis, siue sol supra terras, sicut nox sol sub terris.

Cf. Isidoro, DNR, I, I: Dies est solis orientis praesentia, quousque ad occasum perueniat.

En el cap. VIII: De die del Die diuisionibus temportm, atribuido a Beda se recoge la información de Isidoro, Etym., V, XXX, 1, mientras que nuestros mss. parten de Isidoro, $D N R, \mathrm{I}, \mathrm{I}$.
} 
- Quot ${ }^{9}$ sunt diuisiones diei artificialis 10 ? 11

- Tres: mane et meridies et suprema ${ }^{12}$.

- Quomodo diffinitur ${ }^{13}$ mane uel quot ${ }^{14}$ horas ${ }^{15}$ habet?

- Mane dicitur ab ortu solis usque ad horam ${ }^{16}$ quintam; miridies, ad horam ${ }^{17}$ quintam usque ad horam ${ }^{18}$ nonam; supprema, ab hora ${ }^{19}$ nona usque ad occasum solis.

- Mane ergo quomodo diffinitur? ${ }^{20}$

- Isidorus diffiniuit ${ }^{21}$ dicens: Mane lux matura et plena. Et dictum mane a mana; manum enim antiqui bonum dicebant. Quid enim melius luce? Siue enim mane a Manibus, diis infernorum, qui ros ad augendas fruges distillant, quorum conuersata luna ad terram est. Gentiles enim putabant, quando a nobis sol recedit, quod dies infernorum illum ad se traxissent. Ideo antiqua gentilitas oriente sole colebant.
D. Quot sunt spatia artificialis diei?

M. Tria.

D. Quae?

M. Mane, meridies et suprema.

D. Mane quid est, et quomodo diffinitur, uel quot horas habet?

M. Mane dicitur ab ortu solis usque ad horam post tertiam: meridies, ab hora post tertiam usque ad horam ante nonam: suprema, ab hora ante nonam usque ad occasum solis.

D. Mane ergo quomodo diffinitur?

M. Isidorus diffiniuit dicens: Mane lux matutina et plena, et dictum mane a mano. Manum enim antiqui bonum dicebant. Quid enim melius luce? Propterea ueteres manum diem appellabant, scilicet a puritate. Siue ergo mane dicitur a manibus, id est diis infernorum. Gentiles enim aestimabant, quando sol recedit, ut dii infernorum illum ad se traxissent.

${ }^{9}$ Quod Riu. 59.

10 artificalis Riu. 59. En el ms. Vat. Reg. Lat. 123 una segunda mano, la misma que ha repuntuado y añadido más interrogaciones, corrige la forma articularis por artificalis.

${ }_{11}$ Nótese que nuestros mss. reducen a una sola las dos respuestas que da el magister en la obra atribuida a Beda.

12 supprema Riu. 59.

13 difinitur Riu. 59.

14 quod Riu. 59.

15 oras Riu. 59.

16 oram Riu. 59.

17 oram Riu. 59.

18 oram Riu. 59.

19 ora Riu. 59.

${ }^{20} \mathrm{Cf}$. Isidoro, Etym., V, XXX, 13-14: Mane lux matura et plena, nec iam crepusculum. Et dictum mane a mano; manum enim antiqui bonum dicebant. Quid enim melius luce? Alit mane aestimant uocari a Manibus, quorum conuersatio a luna ad terram est.

Como se puede ver, nuestros mss. siguen con más fidelidad a Isidoro.

21 difiniuit Riu. 59. 
- Meridies quomodo diffinitur ${ }^{22}{ }^{23}$

- Vt Isidorum dixit: Meridies dicta quasi medidies ${ }^{24}$, hoc est medius dies; uel quia tunc purior dies est. Merum enim purum dicitur. In toto enim die nihil ${ }^{25}$ clarius meridie, quando sol e medio caelo rutilat et omnem orbem pari claritate inlustrat.

- Supprema quare dicitur? ${ }^{26}$

- Hoc est suppremendo quia postrema pars diei est, quuando sol ${ }^{27}$ cursum suum in occasum uertit ${ }^{28}$. Hinc dicta quuod superest ad partem ultimum diei ${ }^{29}$. Tunc enim dies ad uesperum ${ }^{30}$ suppremitur quando nubilose noctis caligine obumbratur. Haec ${ }^{31}$ ergo tria spatia ${ }^{32}$ artificalis diei $<$ sunt $>{ }^{33}, a^{34}$ cursu solis temperantur. Mane dicitur ab ortu solis usque dum sol ascenderit in altitudinem caeli. Meridies autem, quando per medium caelum in altitudinem sol occurrit Suppremum, quando sol de altitudine caeli descendit ad occasum.
D. Meridies quomodo diffinitur?

M. Isidorum diffiniuit, dicens: Meridies dicitur quasi purus dies. In toto enim die nihil purius meridie. Antiqui enim merum purum dicebant.

D. Supremum quare dicitur?

M. Quasi supprimendo. Tunc enim sol inclinatur ad occasum. Haec ergo tria spatia artificialis diei sunt, et a cursu solis temperantur. Mane dicitur ab ortu solis usque dum sol ascenderit in altitudinem caeli. Meridies dicitur, quando per medium caelum, in altitudine sol currit. Supremum autem dicitur, quando sol de altitudine caeli descendit ad occasum.

22 difinitur Riu. 59.

${ }^{23} \mathrm{Cf}$. Isidoro, Etym., V, XXX, 15: Meridies dicta quasi medidies, hoc est medius dies; uel quia tunc purior dies est. Merum enim purum dicitur. In toto enim die nihil clarius meridie, quando sol de medio caelo rutilat et omnem orbem pari claritate inlustrat.

El texto de los mss. de Ripoll se muestra más fiel a la fuente: Isidoro.

24 medi dies Vat. Reg. Lat. I23.

25 nichil Riu. 59.

${ }^{26} \mathrm{Cf}$. Isidoro, Etym., V, XXX, 16-17: Suprema est postrema pars diei, quando sol cursum suum in occasum uertit: dicta quod superest ad partem ultimam diei.

Esta definición se omite en la obra atribuida a Beda.

${ }^{27}$ sol om. Riu. 59.

28 uertit om. Riu. 59.

29 diem, raspado en diei Vat. Reg. Lat. 123.

30 uesporum Riu. 59.

31 Hec Riu. 59

32 spacia Vat. Reg. Lat. 123.

33 sunt om. Riu. 59; Vat. Reg. Lat. 123.

34 ac Riu. 59. 
- Quomodo dies naturalis diffinitur? 35

- Vt Isidorus dixit: Dies gemini appellari ${ }^{36}$ solet, proprie ${ }^{37}$ et abusiue ${ }^{38}$; proprie enim a solis ortu, donec rursus oriatur; abusiue a solis ortu, usquequio ${ }^{39}$ ad occasum ${ }^{40}$ perueniat. Et haec ${ }^{41}$ dua spatia ${ }^{42}$ uocantur interdianum et nocturnum, sed interdianum in uetere lege precedebat; nocturnum uero in noua precedit.

- Quot sunt principia naturalis diei? 43

- Quattuor, id est, Caldei et per se ${ }^{44}$ ex ortu solis initium diei dicunt, quia solem colebant. Ebrei ${ }^{45}$ uero et Ateniènses meridie initium sumant ${ }^{46}$, quia secundum lunam numerant; in meridie enim aetas ${ }^{47}$ lunae ${ }^{48}$ mutari putatur. Greci autem et Egyptii ${ }^{49}$ ex initio noc-
D. Quomodo ergo dies naturalis et dies artificialis simul diffinitur? M. Isidorus diffiniuit, dicens: Dies gemine appellari solet; proprie, ab ortu solis, donec rursus oriatur; abusiue, dies ab ortu solis, quousque ad occasum perueniat.

D. Quot sunt ianuae diei?

M. Quatuor. Apud Chaldaeos ab ortu solis usque ad ortum solis. Apud Aegyptios ab occasu solis usque ad occasum solis. Apud Hebraeos ab hora sexta usque ad horam sextam, quia noctem non computabant $\mathrm{He}$ braei. Apud Romanos a media nocte usque ad mediam noctem.

${ }^{35}$ Cf. Isidoro, DNR, I, 1: Dies gemine appellari solet: proprie a solis exortu, donec rursus oriatur, abusiue a solis ortu usquequo ueniat ad occasum. Spatia diei duo sunt, interdianum et nocturnum.

Beda (?) se inspira, en cambio, en isidoro, Etym., V, XXX, 1: Dies legitimus uiginti quattuor horarum...

36 apellari Riu. 59.

37 propriae Vat. Reg. Lat. 123.

38 abusiuae Vat. Reg. Lat. 123.

39 usqueqo Riu. 59.

to ocasum Vat. Reg. Lat. 123.

+1 hec Riu. 59.

42 spacia Riu. 59.

t3 Cf. Isidoro, Etym., V, XXX, 4: Dies secundum Aegyptios inchoat ab occasu solis: secundum Persas ab ortu solis: secundum Athenienses a sexta hora diei; secundum Romanos a media nocte.

Cf. Isidoro, DNR, I, 2: Nam Chaldaei a sole exorto diei initium faciunt, totum id spatium unum diem appellantes. Aegyptii autem ex initio hoctis sequentis diei originem trahunt. Romani autem a medio noctis orivi diem uolunt, et in medio noctis finiri.

44 sae Vat. Reg. Lat. 123.

${ }^{45}$ Haebrei Vat. Reg. Lat. 123.

46 sumunt Vat. Reg. Lat. 123.

47 etas Riu. 59.

48 lune Riu. 59.

49 Egiptii Rill. 59. 
tis initium diei putant, quia lunam sequntur ${ }^{50}$. Romani enim ex medio noctis incipere diem et finire uolunt, sequentes Euangelium dicentem: Media nocte clamor factus est et reliqua. Item secundum Hieronimum: Media nocte factus est mundus, et in media nocte iterum destruetur. Sucundum has ${ }^{51}$ estimationes nox nihil ${ }^{52}$ est sed ${ }^{53}$ absentia lucis ${ }^{54}$.

José Martínez Gázquez y Gemma Puigvert i Planagumà

50 secuntur Riu. 59.

51 has superscriptum inter secundum estimationes Vat. Reg. Lat. 123.

52 nichil Riu. 59.

53 set Riu. 59.

54 Si bien el texto de base del que se parte es el $D e$ die, capitulo VIII de la obra $D e$ diuisionibus temporum atribuida a Beda, las numerosas variaciones observadas entre este texto y nuestros mss. (pensamos, por ejemplo, en las variaciones en el orden de presentación de los argumentos, en la fidelidad con que se sigue a Isidoro) nos conducen a pensar que el copista o los copistas de Ripoll disponían, además del texto atribuido a Beda, de algunas obras de Isidoro, hecho nada extraño habida cuenta que en el inventario de 1047, realizado a la muerte del abad Oliba, figuran las Etymologiae. 